\title{
Synthesis of Vanadium Phosphate Catalysts by Hydrothermal Method for Selective Oxidation of n-butane to Maleic Anhydride
}

\begin{abstract}
Two vanadium phosphate catalysts (VPH1 and VPH2) prepared via hydrothermal method are described and discussed. Both catalysts exhibited only highly crystalline pyrophosphate phase. SEM showed that the morphologies of these catalysts are in plate-like shape and not in the normal rosette-type clusters. Temperature-programmed reduction in $\mathrm{H} 2$ resulted two reduction peaks at high temperature in the range of 600-1100 K. The second reduction peak appeared at $1074 \mathrm{~K}$ occurred as a sharp peak indicated that the oxygen species originated from V4+ phase are having difficulty to be removed and their nature are less reactive compared to other methods of preparation. Modified VPH2 gave better catalytic performance for n-butane oxidation to maleic anhydride contributed by a higher BET surface area, high mobility and reactivity of the lattice oxygen associated to the V4+ which involved in the hydrocarbon's activation. A slight increased of the V5+ phase also enhanced the activity of the VPH2 catalyst.
\end{abstract}

Keyword: vanadium phosphate, hydrothermal, butane oxidation, lattice oxygen 